\title{
ON PARASTATISTICS DEFINED AS TRIPLE OPERATOR ALGEBRAS
}

\author{
Stjepan Meljanac ${ }^{\dagger}$, Marijan Mileković and Marko Stojić $^{\dagger}$ \\ ${ }^{\dagger}$ Rudjer Bošković Institute, Bijenička c.54, 10001 Zagreb, Croatia \\ E-mail: meljanac@thphys.irb.hr \\ $\ddagger$ Prirodoslovno-Matematički Fakultet,Zavod za teorijsku fiziku, \\ Bijenička c.32, 10000 Zagreb, Croatia \\ E-mail: marijan@phy.hr
}

\begin{abstract}
We unify parastatistics, defined as triple operator algebras represented on Fock space, in a simple way using the transition number operators. We express them as a normal ordered expansion of creation and annihilation operators. We discuss several examples of parastatistics, particularly Okubo's and Palev's parastatistics connected to many-body Wigner quantum systems. We relate them to the notion of extended Haldane statistics.
\end{abstract}

PACS No: 05.30.-d, 71.10.+x, 03.65 Bz 


\begin{abstract}
We unify parastatistics, defined as triple operator algebras represented on Fock space, in a simple way using the transition number operators. We express them as a normal ordered expansion of creation and annihilation operators. We discuss several examples of parastatistics, particularly Okubo's and Palev's parastatistics connected to many-body Wigner quantum systems. We relate them to the notion of extended Haldane statistics.
\end{abstract}




\section{Introduction}

Recently, a class of parastatistics (generalizing Bose and Fermi statistics) has been reformulated in terms of Lie supertriple systems ${ }^{1}$. Particularly, Green's parastatistics $^{2}$ as well as new kinds of parastatistics discovered by Palev ${ }^{3,15}$ are reproduced. However, in this approach the positive definite Fock space representations are not treated.

On the other hand, a unified view of all operator algebras represented on Fock spaces has been presented ${ }^{4}$. Furthermore, the permutation invariant statistics are also studied in detail ${ }^{5}$.

Along the lines of Refs. $(4,5)$, in this paper we unify, in a simple way triple operator algebras of Ref.(1), represented on the Fock spaces, as well as Greenberg's infinite quon statistics ${ }^{6}$ and Govorkov's paraquantization ${ }^{7}$. Particularly, we present and discuss parastatistics which naturally appears in many-body Wigner quantum systems ${ }^{3}$ and its (bosonic and supersymmetric) extension ${ }^{15}$. It appears that they are a generalization of Klein-Marshalek algebra ${ }^{8}$, extensively used in nuclear physics. We discuss them in the framework of the Haldane's definition of statistics ${ }^{9}$. We point out that none of them is an example of the original Haldane exclusion statistics, but can be related to the so-called extended Haldane statistics ${ }^{10}$. For each of them we find the extended Haldane statistics parameters . 


\section{Operator algebra, Fock space realization and statistics}

Let us start with any algebra of M pairs of creation and annihilation operators $a_{i}^{\dagger}$, $a_{i}, i=1,2, . . M\left(a_{i}^{\dagger}\right.$ is Hermitian conjugated to $\left.a_{i}\right)$. The algebra is defined by a normally ordered expansion $\Gamma$ (generally no symmetry principle is assumed)

$$
a_{i} a_{j}^{\dagger}=\Gamma_{i j}\left(a^{\dagger} ; a\right)
$$

with the number operators $N_{i}$, i.e., $\left[N_{i}, a_{j}^{\dagger}\right]=a_{i}^{\dagger} \delta_{i j},\left[N_{i}, a_{j}\right]=-a_{i} \delta_{i j}$. In this case no peculiar relations of the type $a_{i}^{m}=a_{j}^{n}, i \neq j$ can appear. Then, every monomial in $\Gamma_{i j}$, Eq.(1), is of the type $\left(\cdots a_{j}^{\dagger} \cdots a_{i} \cdots\right)$ and all other indices appear in pairs $\left(\cdots a_{k}^{\dagger} \cdots a_{k} \cdots\right)$. The corresponding coefficients of expansion can depend on the total number operator $N=\sum_{i=1}^{M} N_{i}$.

We assume that there is a unique vacuum $\mid 0>$ and the corresponding Fock space representation. The scalar product is uniquely defined by $<0 \mid 0>=1$, the vacuum condition $a_{i} \mid 0>=0, i=1,2, . . M$, and Eq.(1). A general N-particle state is a linear combination of the vectors $\left(a_{i_{1}}^{\dagger} \cdots a_{i_{N}}^{\dagger} \mid 0>\right), i_{1}, \cdots i_{N}=1,2, \ldots M$. We consider Fock spaces with no state vectors of negative squared norms. Note that we do not specify any relation between the creation (or annihilation) operators. They appear as a consequnce of the norm zero vectors (null-vectors) in Fock space.

For fixed $\mathrm{N}$ mutually different indices $i_{1}, \cdots i_{N}$, we define the $(N ! \times N !)$ hermitian

matrix of scalar products between states $\left(a_{i_{\pi(1)}^{\dagger}}^{\dagger} \cdots a_{i_{\pi(N)}}^{\dagger} \mid 0>\right)$ for all permutations $\pi \in S_{N}$. The number of linearly independent states among them is given by $d_{i_{1}, \cdots i_{N}}=$ $\operatorname{rank} \mathcal{A}\left(i_{1}, \cdots i_{N}\right)$. The set of $d_{i_{1}, \cdots i_{N}}$ for all possible $i_{1}, \cdots i_{N}$ and all integers $\mathrm{N}$ completely characterizes the statistics and the thermodynamic properties of a free 
system with the corresponding Fock space ${ }^{10}$.

If the algebra (1) is permutation invariant ${ }^{5}$, i.e. $\langle\pi \mu \mid \pi \nu\rangle=\langle\mu \mid \nu\rangle$, for all $\pi, \mu, \nu \in$ $S_{N}$, all expansion terms in $\Gamma_{i j}$ of the form (symbolically)

$$
\Gamma_{i j}:=\sum(\underbrace{a^{\dagger} \cdots a^{\dagger}}_{l-r})(a_{j}^{\dagger} \underbrace{a^{\dagger} \cdots a^{\dagger}}_{r} \underbrace{a \cdots a}_{s} a_{i})(\underbrace{a \cdots a}_{l-s})
$$

have the same coefficient for all $i, j=1,2, \ldots M$. ( One single relation, for example $a_{1} a_{2}^{\dagger}=\Gamma_{12}$, determines the whole algebra.)

For the permutation invariant algebras there are several important consequences ${ }^{5}$.

\section{Consequences}

(i) The matrices $\mathcal{A}\left(i_{1}, \cdots i_{N}\right)$ and their ranks do not depend on concrete indices $i_{1}, \cdots i_{N}$, but only on the multiplicities $\lambda_{i}$ of appearance of the same indices $\lambda_{1} \geq \lambda_{2} \geq \ldots \geq \lambda_{M} \geq 0,|\lambda|=\sum_{i=1}^{M} \lambda_{i}=N$, i.e. on the partition $\lambda$ of $N$.

(ii) For mutually different indices $i_{1}, \cdots i_{N}$ i.e. $\lambda_{1}=\lambda_{2}=\cdots \lambda_{N}=1$, the generic matrix $\mathcal{A}_{1^{N}}$ is

$$
\mathcal{A}_{1^{N}}=\sum_{\pi \in S_{N}} c(\pi) R(\pi)
$$

where $\mathrm{R}$ is the right regular representation of the permutation group $S_{N}$ and $c(\pi)$ are (real) coefficients. In other words, any row (column) of the matrix determines the whole matrix $\mathcal{A}_{1^{N}}$.

(iii) All matrices $\mathcal{A}_{\lambda}$ can be simply obtained from $\mathcal{A}_{1^{N}}{ }^{5}$. To check that the Fock space does not contain states of negative norms, it is sufficient to show that only generic matrices are non-negative ${ }^{11}$.

(iv) For permutation invariant algebras there exist the transition number operators $N_{i j}, i, j=1,2 \ldots M$ with the properties 


$$
\left[N_{i j}, a_{k}^{\dagger}\right]=\delta_{i k} a_{j}^{\dagger}, \quad\left[N_{i j}, a_{k}\right]=-\delta_{j k} a_{i}, \quad N_{i j}^{\dagger}=N_{j i}, \quad N_{i i} \equiv N_{i}
$$

$N_{i j}$ can be presented similarly as $\Gamma_{i j}$, i.e. as a normal ordered expansion

$$
N_{i j}=a_{j}^{\dagger} a_{i}+\alpha \sum_{l} a_{l}^{\dagger} a_{j}^{\dagger} a_{i} a_{l}+\beta \sum_{l}\left(a_{l}^{\dagger} a_{j}^{\dagger} a_{l} a_{i}+a_{j}^{\dagger} a_{l}^{\dagger} a_{i} a_{l}\right)+\gamma \sum_{l} a_{j}^{\dagger} a_{l}^{\dagger} a_{l} a_{i}+\cdots
$$

where $\alpha, \beta, \gamma$ are constants which do not depend on the indices $i, j$.

In the next section we show that all permutation invariant statistics considered by Okubo ${ }^{1}$, Palev ${ }^{3,15}$, Greenberg ${ }^{6}$, Govorkov ${ }^{7}$ and Klein and Marshalek ${ }^{8}$ can be simply unified in terms of triple-operator algebras

$$
\left[\left[a_{i}, a_{j}^{\dagger}\right]_{q}, a_{k}^{\dagger}\right]=x \delta_{i j} a_{k}^{\dagger}+y \delta_{i k} a_{j}^{\dagger}+z \delta_{j k} a_{i}^{\dagger}
$$

for all $i, j, k=1,2, \cdots M$. Here, $x, y, z \in \mathbf{R}$ are constants, [, ] denotes the commutator and $[a, b]_{q}=a b-q b a$ is the q-deformed commutator.

\section{Remarks}

1. Equation (1), together with the vacuum condition $a_{i} \mid 0>=0$, uniquely determines all matrices $\mathcal{A}_{1^{N}}$ and $\mathcal{A}_{\lambda}$. However, equation (1) does not imply positive definiteness, which has to be checked separately.

2. All other triple-operator relations follow from Eq.(5) via hermiticity of creation and annihilation operators, a linear combination of Eq.(5) with indices interchanged and, finally,as null-states of matrices $\mathcal{A}_{\lambda}$.

3. The algebra with the well defined number operators $N_{i}$ imply that $z=0$ in (5). If $z \neq 0$, there exist peculiar relations of the type $a_{i}^{2}=a_{j}^{2}$ for all $i, j=1,2, \cdots M$, 
although $a_{i}^{\dagger}|0\rangle$ are linearly independent states. Such peculiar algebras are consistent if the Fock space does not contain null-states ${ }^{12}$.

4. We point out that the algebra (5) can be simply written as the normal ordered expansion

$$
a_{i} a_{j}^{\dagger}=(1+x N) \delta_{i j}+q a_{j}^{\dagger} a_{i}+y N_{i j}+z N_{j i}
$$

where $N_{i j}$ are the transition number operator of form $(3,4)$ and $\mathrm{N}$ is the total number operator.

\section{Examples}

Example 1. Green's parastatistics ${ }^{2}$ can be presented in the form of Eq.(6) with $x=z=0, y=\frac{2}{p}, p \in \mathbf{N}$ and $q= \pm 1$, i.e.

$$
a_{i} a_{j}^{\dagger}=\delta_{i j} \mp a_{j}^{\dagger} a_{i} \pm \frac{2}{p} N_{i j}
$$

where the upper (lower) sign corresponds to para-Bose (para-Fermi) statistics.

The transition number operator $N_{i j}$ is, up to the second order, given by ${ }^{4}$

$$
N_{i j}=a_{j}^{\dagger} a_{i}+\frac{p^{2}}{4(p-1)} \sum_{l}\left[Y_{j l}\right]^{\dagger}\left[Y_{i l}\right]+\cdots,
$$

where $Y_{i l}=a_{i} a_{l}-q\left(\frac{2}{p}-1\right) a_{l} a_{i}$.

Example 2. Govorkov's new paraquantization ${ }^{7}$ is given by $x=z=0$, $y=\frac{\lambda}{p}, \lambda= \pm 1, p \in \mathbf{N}$ and $q=0:$

$$
a_{i} a_{j}^{\dagger}=\delta_{i j}-\frac{\lambda}{p} N_{i j}
$$


with the transition number operator, up to the second order,

$$
N_{i j}=a_{j}^{\dagger} a_{i}+\frac{p^{2}}{p^{2}-\lambda^{2}} \sum_{l}\left[Y_{j l}\right]^{\dagger}\left[Y_{i l}\right]+\cdots,
$$

where $Y_{i l}=a_{i} a_{l}+\left(\frac{\lambda}{p}\right) a_{l} a_{i}$.

Example 3. Greenberg's infinite quon statistics ${ }^{6}$ is given by $x=y=z=0$ and $-1<q<1$ :

$$
a_{i} a_{j}^{\dagger}=\delta_{i j}+q a_{j}^{\dagger} a_{i}
$$

with transition number operator, up to the second order,

$$
N_{i j}=a_{j}^{\dagger} a_{i}+\frac{1}{1-q^{2}} \sum_{l}\left[Y_{j l}\right]^{\dagger}\left[Y_{i l}\right]+\cdots,
$$

where $Y_{i l}=a_{i} a_{l}-q a_{l} a_{i}$. (The closed form for $N_{i j}$ to all orders and for the general parameter $q_{i j}$ is presented in Ref.(13).)

Example 4. Palev's A statistics (Fermi case), which appears naturally in the treatment of many-body Wigner quantum systems ${ }^{3}$, is described by the following algebra $(i, j, k=1,2, \cdots M)$ :

$$
\begin{gathered}
{\left[\left\{a_{i}, a_{j}^{\dagger}\right\}, a_{k}^{\dagger}\right]=\delta_{i k} a_{j}^{\dagger}-\delta_{i j} a_{k}^{\dagger}} \\
{\left[\left\{a_{i}, a_{j}^{\dagger}\right\}, a_{k}\right]=-\delta_{j k} a_{i}+\delta_{i j} a_{k}} \\
\left\{a_{i}, a_{j}\right\}=\left\{a_{i}^{\dagger}, a_{j}^{\dagger}\right\}=0 .
\end{gathered}
$$

Hereafter, $\{$,$\} denotes the anticommutator.$

(In the original algebra, the operators depend on two indices, $a_{i} \mapsto a_{\alpha i}$, but the structure of the algebra depends on the single index. One recovers the original algebra with $\left.\delta_{\alpha i, \beta j}=\delta_{\alpha \beta} \delta_{i j}\right)$. The vacuum conditions are $a_{i}|0\rangle=0, a_{i} a_{j}^{\dagger}|0\rangle=p \delta_{i j}|0\rangle$ 
for $p \in \mathbf{N}$. Upon the redefinition of the operators $\left(a_{i}, a_{i}^{\dagger}\right) \mapsto\left(\sqrt{p} a_{i}, \sqrt{p} a_{i}^{\dagger}\right)$, we write the above algebra as normal ordered expansion with $x=-\frac{1}{p}, y=\frac{1}{p}, z=0$ and $q=-1$

$$
a_{i} a_{j}^{\dagger}=\left(1-\frac{N}{p}\right) \delta_{i j}-a_{j}^{\dagger} a_{i}+\frac{1}{p} N_{i j}
$$

The action of the annihilation operators $a_{i}$ on the Fock states is obtained from the above relation, Eq.(14). For example,

$$
a_{i} a_{j}^{\dagger} a_{k}^{\dagger}|0\rangle=\left(1-\frac{1}{p}\right)\left(\delta_{i j} a_{k}^{\dagger}-\delta_{i k} a_{j}^{\dagger}\right)|0\rangle
$$

It follows that

$$
\begin{gathered}
a_{i}\left(a_{i}^{\dagger}\right)^{2}|0\rangle=0, \quad \forall i \\
a_{i} a_{i}^{\dagger} a_{k}^{\dagger}|0\rangle=-a_{i} a_{k}^{\dagger} a_{i}^{\dagger}|0\rangle=\left(1-\frac{1}{p}\right) a_{k}^{\dagger}|0\rangle, \quad i \neq k .
\end{gathered}
$$

Hence, we obtain $\left\{a_{i}, a_{j}\right\}=\left\{a_{i}^{\dagger}, a_{j}^{\dagger}\right\}=0$.

Generally, for mutually different indices $i_{1}, \cdots i_{N}$, we find

$$
a_{i_{1}} a_{i_{1}}^{\dagger} a_{i_{2}}^{\dagger} \cdots a_{i_{N}}^{\dagger}|0\rangle=\left(1-\frac{N-1}{p}\right) a_{i_{2}}^{\dagger} \cdots a_{i_{N}}^{\dagger}|0\rangle
$$

in accordance with Ref.(3). The Fock space does not contain negative norm states if $p \in \mathbf{N}$. The above equation (16) implies that the allowed states are only those with $N \leq p$, and the states with $N>p$ are null-states.

The transition number operartor $N_{i j}$, up to the second order, is:

$$
N_{i j}=a_{j}^{\dagger} a_{i}+\frac{1}{(p-1)} \sum_{l} a_{l}^{\dagger} a_{j}^{\dagger} a_{i} a_{l}+\frac{2}{(p-1)(p-2)} \sum_{l_{1}, l_{2}} a_{l_{2}}^{\dagger} a_{l_{1}}^{\dagger} a_{j}^{\dagger} a_{i} a_{l_{1}} a_{l_{2}}+\cdots
$$

and terminates with $p$ creation and $p$ annihilation operator terms. For example, if $p=2$, the terms with $(p-2)$ appearing in the denominator do not appear at all. 
The $p \rightarrow \infty$ reproduces the Fermi algebra. We note that case $p=1$ reproduces the Klein-Marshalek algebra ${ }^{8}$, namely only the one-particle states are allowed:

$$
a_{i} a_{j}^{\dagger}=(1-N) \delta_{i j}, \quad N=\sum_{l} a_{l}^{\dagger} a_{l} .
$$

In this sense, algebra (14) generalizes the Klein-Marshalek algebra.

It is interesting that the Fock space generated by the algebra (14) is equivalent to the Fock space generated by the algebra (with the same vacuum condition imposed)

$$
a_{i} a_{j}^{\dagger}=\left(1-\frac{N}{p}\right)\left(\delta_{i j}-a_{j}^{\dagger} a_{i}\right)
$$

with the same $N_{i j}$ and $N$ as given by Eq.(17).

Furthermore, there are infinitely many algebras leading to different generic matrices, but with the same statistics. They can be represented by

$$
a_{i} a_{j}^{\dagger}=f(N)\left(\delta_{i j}-a_{j}^{\dagger} a_{i}\right)
$$

with $f(n)>0, n<p$ and $f(p)=0$. The simplest choice is the step function $f(N)=\Theta(p-N)(\Theta(x)=0, x \leq 0$ and $\Theta(x)=1, x>0)$.

We point out that the corresponding statistics is Fermi statistics restricted up to $N \leq p$ N-particle states. Hence, the counting rule is simply $D^{F}(M, N)=\left(\begin{array}{c}M \\ N\end{array}\right)$, $N \leq p$ and $D^{F}(M, N)=0$ if $N>p$. Recall that Haldane ${ }^{9}$ introduced the statistics parameter $g$ through the change of the single-particle Hilbert space dimension $d_{n}$

$$
g_{n \rightarrow n+\Delta n}=\frac{d_{n}-d_{n+\Delta n}}{\Delta n},
$$

where $n$ is the number of particles and $d_{n}$ is the dimension of the one-particle Hilbert space obtained by keeping the quantum numbers of $(n-1)$ particles fixed. In the 
similar way we define the extended statistics parameter through the change of the available one-particle Fock-subspace dimension ${ }^{10}$. Therefore, the above statistics is characterized by the Haldane statistical parameter $g=1$

$$
g_{n \rightarrow n+k}=\frac{d_{n}-d_{n+k}}{k}=\frac{(M-n+1)-(M-n-k+1)}{k}=1,
$$

if $n+k \leq p$. If $n+k=p+1$, then $g_{n \rightarrow n+k}=\frac{(M-n+1)}{(p-n+1)}, n=1,2, \cdots p$ is fractional but $g$ is not constant any more. Hence, this is not an example for the original Haldane statistics for which the statistics parameter is $g=$ const. Moreover, the above statistics is also not the statistics of the Karabali-Nair type ${ }^{14}$, where $a_{i}^{p} \neq 0$, $a_{i}^{p+1}=0$, and for any $N \leq M p$ N-particle state is allowed, since from the Eq.(15) we already have $a_{i}^{2}=0$ and $N \leq p$.

Example 5. Palev's A statistics ${ }^{15}$ (Bose case) is the counterpart of the algebra (14), namely:

$$
\begin{gathered}
{\left[\left[a_{i}, a_{j}^{\dagger}\right], a_{k}^{\dagger}\right]=-\delta_{i k} a_{j}^{\dagger}-\delta_{i j} a_{k}^{\dagger},} \\
{\left[\left[a_{i}, a_{j}^{\dagger}\right], a_{k}\right]=\delta_{j k} a_{i}+\delta_{i j} a_{k},} \\
{\left[a_{i}, a_{j}\right]=\left[a_{i}^{\dagger}, a_{j}^{\dagger}\right]=0, \quad i, j, k=1,2, \cdots M .}
\end{gathered}
$$

and the vacuum condition $a_{i} a_{j}^{\dagger}|0\rangle=p \delta_{i j}|0\rangle$. After the redefinition of the operators $\left(a_{i}, a_{i}^{\dagger}\right) \mapsto\left(\sqrt{p} a_{i}, \sqrt{p} a_{i}^{\dagger}\right)$, we write the normal ordered expansion of $a_{i} a_{j}^{\dagger}$ as $\left(x=y=-\frac{1}{p}, z=0, q=-1\right)$

$$
a_{i} a_{j}^{\dagger}=\left(1-\frac{N}{p}\right) \delta_{i j}+a_{j}^{\dagger} a_{i}-\frac{1}{p} N_{i j}
$$

The action of the annihilation operators $a_{i}$ on the Fock states is obtained from 
Eq.(23). For example,

$$
a_{i} a_{j}^{\dagger} a_{k}^{\dagger}|0\rangle=\left(1-\frac{1}{p}\right)\left(\delta_{i j} a_{k}^{\dagger}+\delta_{i k} a_{j}^{\dagger}\right)|0\rangle
$$

Hence, we obtain $\left[a_{i}, a_{j}\right]=\left[a_{i}^{\dagger}, a_{j}^{\dagger}\right]=0$.

Generally, we find

$$
\left.a_{i}\left(a_{1}^{\dagger}\right)^{n_{1}}\left(a_{2}^{\dagger}\right)^{n_{2}} \cdots\left(a_{M}^{\dagger}\right)^{n_{M}}|0\rangle=N_{i}\left(1-\frac{N-1}{p}\right)\left(a_{1}^{\dagger}\right)^{n_{1}}\left(a_{2}^{\dagger}\right)^{n_{2}} \cdots\left(a_{i}^{\dagger}\right)^{n_{i}-1} \cdots a_{M}^{\dagger}\right)^{n_{M}}|0\rangle
$$

where $N=\sum_{i=1}^{M} n_{i}$. The Fock space does not contain negative norm states if $p \in \mathbf{N}$. The above equation (24) implies that the states with $N \leq p$ are allowed and the states with $N>p$ are null-states. The transition number operator $N_{i j}$ has the same form, with the same coefficients as in the Fermi case, Eq.(17), and terminates with p-annihilation and p-creation operator terms. The limit $p \rightarrow \infty$ reproduces the Bose algebra. We note that if $p=1$ the above algebra (23) reproduces the Klein-Marshalek algebra ${ }^{8}$. Hence, this algebra is the Bose generalization of the Klein-Marshalek algebra.

There are again infinitely many algebras leading to different generic matrices but of the same ranks, i.e. statistics. They can be represented by

$$
a_{i} a_{j}^{\dagger}=f(N)\left(\delta_{i j}+a_{j}^{\dagger} a_{i}\right)
$$

with $f(n)>0, n<p$ and $f(p)=0$. The simplest choice is the step function mentioned after Eq. (20) or $f(N)=1-\frac{N}{p}$. The corresponding statistics is Bose statistics restricted to $\mathrm{N}$-particle states with $N \leq p$. Hence, the counting rule is simply $D^{B}(M, N)=\left(\begin{array}{c}M+N-1 \\ N\end{array}\right), N \leq p$ and $D^{B}(M, N)=0$ if $N>p$. Therefore, the above statistics is characterized by the Haldane statistics parameter $g=0$ 


$$
g_{n \rightarrow n+k}=\frac{d_{n}-d_{n+k}}{k}=\frac{M-M}{k}=0,
$$

if $n+k \leq p$. If $n+k=p+1$, then $g_{n \rightarrow n+k}=\frac{M}{(p-n+1)}, n=1,2, \cdots p$, is fractional but not constant . Hence, this is not an example for the original Haldane exclusion statistics for which $g$ should be constant. The above statistics is also not of the Karabali-Nair type ${ }^{14}$, since $a_{i}^{p} \neq 0, a_{i}^{p+1}=0$ but $N \leq p$. This would be equivalent only for the single-mode oscillator, $M=1$.

Example 6. The Bose and Fermi restricted algebra of Refs. (1,3) (the super-triple system) can be defined as

$$
\begin{gathered}
{\left[a_{I}, a_{J}^{\dagger}\right]_{q}=\left(1-\frac{N}{p}\right) \delta_{I J}-\frac{(-)^{\sigma(I) \sigma(J)}}{p} N_{I J},} \\
q=(-)^{\sigma(I) \sigma(J)}, \\
\sigma(I)=\left\{\begin{array}{lll}
0 & \text { if } I=i & \text { (Bose) } \\
1 & \text { if } I=\alpha & \text { (Fermi) }
\end{array}\right.
\end{gathered}
$$

where the index $I \doteq\left(i=1,2, \cdots M_{B} ; \alpha=1,2, \cdots M_{F}\right.$.) denotes bosonic (fermionic) oscillator and $N=N_{B}+N_{F}$ is the total number operator.

Explicitly,

$$
\begin{gathered}
{\left[a_{i}, a_{j}^{\dagger}\right]=\left(1-\frac{N}{p}\right) \delta_{i j}-\frac{1}{p} N_{i j},} \\
\left\{a_{\alpha}, a_{\beta}^{\dagger}\right\}=\left(1-\frac{N}{p}\right) \delta_{\alpha \beta}+\frac{1}{p} N_{\alpha \beta} \\
{\left[a_{i}, a_{\alpha}^{\dagger}\right]=-\frac{1}{p} N_{i \alpha}} \\
{\left[a_{\alpha}, a_{i}^{\dagger}\right]=-\frac{1}{p} N_{\alpha i} .}
\end{gathered}
$$

The consistency condition for the algebra (27) reads: 


$$
N_{I J} a_{K}^{\dagger}-(-)^{(\sigma(I)+\sigma(J)) \sigma(K)} a_{K}^{\dagger} N_{I J}=\delta_{I K} a_{J}^{\dagger}
$$

For example,

$$
\begin{gathered}
\left\{N_{i \alpha}, a_{\gamma}\right\}=+\delta_{\alpha \gamma} a_{i}, \\
{\left[N_{i \alpha}, a_{j}^{\dagger}\right]=\delta_{i j} a_{\alpha}^{\dagger} .}
\end{gathered}
$$

Notice that $\left(N_{i \alpha}\right)^{2}=0$. Thus, $N_{i \alpha}$ plays the role of supersymmetric charge. Furthermore, it follows that

$$
\left[a_{i}, a_{j}\right]=\left\{a_{\alpha}, a_{\beta}\right\}=\left[a_{i}, a_{\alpha}\right]=0
$$

The action of the annihilation operators $a_{i}, a_{\alpha}$ on the Fock states is obtained by combining Eqs.(27) and (28). The N-particle states are allowed only if $N \leq p$, with $p$ being an integer.

The transition number operators, up to the second order, are basically similar to (17) and read

$$
\begin{gathered}
N_{I J}=a_{J}^{\dagger} a_{I}+\frac{1}{(p-1)} \sum_{L}(-)^{\sigma(L)(\sigma(I)+\sigma(J))} a_{L}^{\dagger} a_{J}^{\dagger} a_{I} a_{L}+ \\
+\frac{2}{(p-1)(p-2)} \sum_{L_{1}, L_{2}}(-)^{\left(\sigma\left(L_{1}\right)+\sigma\left(L_{2}\right)\right)(\sigma(I)+\sigma(J))} a_{L_{2}}^{\dagger} a_{L_{1}}^{\dagger} a_{J}^{\dagger} a_{I} a_{L_{1}} a_{L_{2}}+\cdots,
\end{gathered}
$$

where the sum over $L$ runs over bosonic $\left(i=1,2, \cdots M_{B}\right)$ and fermionic $\left(\alpha=1,2, \cdots M_{F}\right)$ indices.

In the limit $p \rightarrow \infty$, the above algebra reduces to the ordinary Bose and Fermi algebra. If $p=1$, the above algebra reduces to the Klein - Marshalek algebra with $M_{B}+M_{F}$ oscillators. 
Example \%. Okubo's triple operator algebra (Example 4. in Ref.(1)) is defined for the fermionic operators $a_{i}$ as

$$
\left[\left\{a_{i}, a_{j}^{\dagger}\right\}, a_{k}^{\dagger}\right]=\left(\frac{2}{p}\right)\left(-\delta_{i j} a_{k}^{\dagger}-\delta_{j k} a_{i}^{\dagger}+\delta_{i k} a_{j}^{\dagger}\right)
$$

The normal ordered expansion of $a_{i} a_{j}^{\dagger}$ is given by $\left(x=z=-\frac{2}{p}, y=\frac{2}{p}, q=-1\right)$

$$
a_{i} a_{j}^{\dagger}=\left(1-\frac{2 N}{p}\right) \delta_{i j}-a_{j}^{\dagger} a_{i}+\left(\frac{2}{p}\right)\left(N_{i j}-N_{j i}\right) .
$$

In the limit $p \rightarrow \infty$, it becomes the Fermi algebra.

From (31) it follows that

$$
\begin{gathered}
a_{i}\left(a_{j}^{\dagger}\right)^{2}|0\rangle=-\left(\frac{2}{p}\right) a_{i}^{\dagger}|0\rangle, \quad \forall i, j \\
a_{i} a_{i}^{\dagger} a_{k}^{\dagger}|0\rangle=-a_{i} a_{k}^{\dagger} a_{i}^{\dagger}|0\rangle=\left(1-\frac{2}{p}\right) a_{k}^{\dagger}|0\rangle \quad i \neq k .
\end{gathered}
$$

Therefore,

$$
\begin{gathered}
\left\{a_{i}, a_{j}\right\}=\left\{a_{i}^{\dagger}, a_{j}^{\dagger}\right\}=0, \quad i \neq j, \\
\left(a_{i}\right)^{2}=A, \quad\left[a_{i}, A\right]=0, \quad\left[a_{i}, A^{\dagger}\right]=-\left(\frac{2}{p}\right) a_{i}^{\dagger}, \quad \forall i \\
\left(a_{i}\right)^{p} \neq 0, \quad\left(a_{i}\right)^{p+1}=0 .
\end{gathered}
$$

However, in the Fock space there are negative norm states since $\left\langle 0\left|\left(a_{i}\right)^{2}\left(a_{i}^{\dagger}\right)^{2}\right| 0\right\rangle=$ $-\left(\frac{2}{p}\right)<0$. The necessary condition for absence of such states is $z \geq 0$. The algebra similar to the algebra described by Eqs.(31-33) but with the positive definite Fock representations has been called peculiar algebra and was studied in Ref.(12).

Finally, let us mention that all Lie (super) algebras are triple systems ( since $\left.\left[a_{i}, a_{j}^{\dagger}\right]_{ \pm}=\delta_{i j}\left(c_{i}+d_{i} N_{i}\right)\right)$ and for a irreducible representations characterized with highest (lowest) weight state $\Lambda$ ("vacuum") one can find the following normal ordered expansion

$$
a_{i} a_{i}^{\dagger}=\Gamma_{i}\left(a^{\dagger}, a ; \Lambda\right), \quad a_{i} a_{j}^{\dagger}= \pm a_{j}^{\dagger} a_{i}
$$

However, these systems are not permutation invariant in the sense we defined in this paper. 


\section{Acknowledgement}

We thank T.Palev for providing us with references $(3,15)$ and interesting remarks. 


\section{References}

1. S.Okubo, J.Math.Phys.35, 2785 (1994); "Super-triple systems and applications to parastatistics and Yang-Baxter equations" (hep-th/9306160).

2. H.S.Green, Phys.Rev.90, 170 (1953); Y.Ohnuki and S.Kamefuchi, Quantum field theory and parastatistics, University of Tokio Press, Tokio, Springer, Berlin, 1982.

3. T.Palev, Czech. Journ. Phys. B29, 91 (1979); ibid. B32, 680 (1982); J.Math.Phys.23, 1778 (1982); T.Palev and S.Stoilova, J. Phys.A :Math.Gen.27, 977 (1994); ibid. 27,7387 (1994); J.Math.Phys.38, 2806 (1997).

4. S.Meljanac and M.Mileković, Int.J.Mod.Phys.A11, 1391 (1996).

5. B.Melić and S.Meljanac, Phys.Lett.A226 , 22 (1997); S.Meljanac, M.Stojić and D.Svrtan, Phys.Lett.A224, 319 (1997).

6. O.W.Greenberg, Phys. Rev.D43, 4111 (1991); Phys.Rev.Lett.64, 705 (1990).

7. A.B.Govorkov, Theor.Math.Phys.98, 107 (1994); Nucl.Phys.B 365, 381 (1991).

8. A.Klein and E.R.Marshalek, Rev.Mod.Phys.63, 375 (1991); Z.Phys.A329, 441 (1988).

9. F.D.M.Haldane, Phys.Rev.Lett.67, 937 (1991).

10. S.Meljanac and M.Mileković, Mod.Phys.Lett.A11, 3081 (1996).

11. D.Zagier, Comm.Math.Phys.147, 199 (1992); S.Meljanac and D.Svrtan, Comm.Math. 1, 1 (1996) and preprint IRB-TH-5/95. 
12. S.Meljanac, M.Mileković and A.Perica, Europhys.Lett.33, 175 (1996); S.Meljanac, M.Mileković and A.Perica, Int.J.Theor.Phys. 36, 11 (1997).

13. S.Meljanac and A.Perica, J.Phys.A :Math.Gen.27, 4737 (1994); Mod.Phys.Lett.A9, 3293 (1994).

14. D.Karabali and V.P.Nair, Nucl.Phys.B 438, 551 (1995).

15. T.Palev,Habilitation Thesis, Sofia 1976; "Lie algebraic aspect of quantum statistics. Unitary quantization (A-statistics) (hep-th/9705032). 